\title{
Hemodiafiltración en línea pre-dilucional, frente a post- dilucional: estudio comparativo de eficacia dialítica y tolerancia hemodinámica
}

\author{
"Premio Fresenius Medical Care para enfermería, hemodiafiltración en línea 2011"
}

Raquel Menezo Viadero - Mirian García Martínez - Raquel Pelayo Alonso - José Luis Cobo Sánchez Marina Rojo Tordable - Alicia Tovar Rincón - Violeta Olalla Antolín - Araceli Sáenz de Buruaga Perea Hortensia Cepa García - M ${ }^{a}$ Elena Incera Setién - Ana Isabel Pérez Garmilla - Salvadora Peiró Sampayo Carmen Higuera Roldán - Rosa Alonso Nates

Enfermeras/os. Servicio de Nefrología. Hospital Universitario Marqués de Valdecilla. Santander

\section{Resumen}

La hemodiafiltración en línea es una técnica de diálisis que combina las ventajas de la hemodiálisis de alto flujo (transporte difusivo) y la hemofiltración (transporte convectivo).

Esta técnica permite varias alternativas según se incorpore el líquido de reinfusión: pre-dilucional (antes del dializador) y post-dilucional (después del dializador), presentando cada una de ellas ventajas e inconvenientes.

El objetivo de este estudio fue comparar distintos parámetros dialíticos y hemodinámicos entre los modos de hemodiafiltración pre y postdilucional.

Se realizó un estudio prospectivo transversal en una población en diálisis que ya se trataba con hemodiafiltración en línea, sometiéndose durante 4 semanas a cada una de las modalidades (pre y post-dilucional).

Correspondencia:

Raquel Menezo Viadero

Servicio de Nefrología

Hosp. U. Marqués de Valdecilla

Avda. Valdecilla s/n 39008 Santander. Cantabria

E-mail: raquelmevi@gmail.com
Se registraron los valores de: tensión arterial sistólica, diastólica y frecuencia cardiaca pre y post sesión, flujo de sangre, presión venosa, volumen de sangre dializado y volumen de sustitución.

La dosis de diálisis se midió mediante dialisancia iónica.

Se estudiaron 26 pacientes: $30 \%$ mujeres y $70 \%$ hombres, con una edad media de $61 \pm 13$ años. El tiempo medio en tratamiento renal sustitutivo fue de $117 \pm 124,45$ meses, tiempo medio en hemodiálisis fue de $50 \pm 54,38$ meses.

Los parámetros hemodinámicos no han presentado diferencias significativas entre las dos modalidades estudiadas (pre y post-dilucional). Se obtiene un mayor valor de KT estadísticamente significativo en la técnica de hemodiafiltración post-dilucional precisando ésta un volumen de sustitución de la mitad, en comparación con la modalidad predilucional.

\section{Conclusiones:}

La técnica de hemodiafiltración en línea es bien tolerada en las dos modalidades de infusión.

Ante un mismo flujo de sangre, presión venosa y volumen de sangre dializado se consigue una mejor dosis de diálisis en el modo post-dilucional. 
La hemodiafiltración post-dilucional parece ser una mejor alternativa respecto a la pre-dilucional en relación a los parámetros estudiados siempre que no se tenga en cuenta la problemática de los accesos vasculares. Con dicha técnica logramos mejores resultados de $\mathrm{KT}$ precisando la mitad de volumen de sustitución, con el consiguiente ahorro de agua ultrapura.

La hemodiafiltración pre-dilucional puede ser una alternativa para aquellos pacientes que no se consiga un flujo arterial alto.

\section{PALABRAS CLAVE:}

- HEMODIAFILTRACIÓN

- MODALIDADES DE REINFUSIÓN

- DOSIS DE DIÁLISIS

- TOLERANCIA HEMODINÁMICA

\section{Pre-dilution versus post-dilution on-line hae- modiafiltration: a comparative study of dialytic efficacy and haemodynamic tolerance}

\section{Abstract}

On-line haemodiafiltration is a dialysis technique which combines the advantages of high-flow haemodialysis (diffusive transport) and haemofiltration (convective transport).

This technique allows different alternatives depending on how the reinfusion liquid is added: pre-dilution (before the dialyser) and post-dilution (after the dialyser), each of them having advantages and disadvantages.

The object of this study was to compare different dialytic and haemodynamic parameters in the preand post-dilution haemodiafiltration modes.

A transversal prospective study was conducted of a population in dialysis already being treated with on-line haemodiafiltration, using each of the modes (pre- and post-dilution) with them for 4 weeks.
The following values were recorded: systolic and diastolic arterial pressure and cardiac frequency pre- and post-session, blood flow, venous pressure, volume of blood dialysed and replacement volume.

Dialysis dosage was measured by means of ionic dialysance.

26 patients were studied: $30 \%$ women and $70 \%$ men, with an average age of $61 \pm 13$ years. The average time under renal replacement treatment was $117 \pm 124.45$ months, and the average time in haemodialysis was $50 \pm 54.38$ months.

The haemodynamic parameters showed no significant differences between the two modes studied (preand post-dilution). A statistically significant higher value for $\mathrm{KT}$ was obtained for the post-dilution haemodiafiltration technique, requiring half the replacement volume of the pre-dilution mode.

\section{Conclusions:}

The on-line haemodiafiltration technique is tolerated well in both infusion modes.

With the same blood flow, venous pressure and volume of blood dialysed, a higher dialysis dosage was achieved using the post-dilution mode.

Post-dilution haemodiafiltration appears to be a better alternative than the pre-dilution mode in terms of the parameters studied as long as the issue of vascular access is not taken into account. Better KT results were achieved with this technique, requiring half the replacement volume, with the resulting saving in ultra-pure water.

Pre-dilution haemodiafiltration may be an alternative for patients where a high arterial flow cannot be achieved.

\section{KEY WORDS:}

- HAEMODIAFILTRATION

- METHODS OF REINFUSION

- DIALYSIS DOSE

- HAEMODYNAMIC TOLERANCE 


\section{Introducción}

La hemodiafiltración (HDF) en-linea es una técnica de diálisis que combina las ventajas de la hemodiálisis de alto flujo (transporte difusivo) y la hemofiltración (transporte convectivo). La característica que la diferencia de la hemodiafiltración convencional es que utiliza un líquido de sustitución, para asegurar un volumen convectivo eficaz, a partir del líquido de diálisis tras el paso de éste por dos filtros de polisulfona que atrapan posibles endotoxinas o pirógenos, produciendo un líquido de reinfusión "cuasi" estéril.

Esta técnica precisa de dializadores de alto flujo, membranas de alta biocompatibilidad, vigilancia minuciosa y continua del agua que garantice un líquido de diálisis ultrapuro y altos volúmenes de reposición ${ }^{1,2,3}$.

Las ventajas de la HDF son:

- Mejor depuración de todo tipo de moléculas presentes en el suero del paciente urémico, lo que conduce a un descenso de la morbilidad.

- Mejor control de la anemia y del estado nutricional por la mayor eficacia depurativa y la alta pureza del agua empleada.

- Estabilidad cardiovascular durante el tratamiento ${ }^{4}$.

La HDF permite varias alternativas según se incorpore el líquido de reinfusión: pre-dilucional (antes del dializador), post-dilucional (después del dializador) y mild-dilucional (la reinfusión se realiza de forma mixta entre pre-dilucional y post-dilucional).

La HDF pre-dilucional, respecto a la hemodiálisis de alto flujo presenta unos aclaramientos más bajos de solutos de pequeño peso molecular, aunque permite una mejor extracción de moléculas de mediano y alto peso molecular. Precisa de elevados flujos de infusión y reduce la hemoconcentración. Está indicada en los casos en los que el paciente tenga hematocritos altos 0 en los que el acceso vascular no pueda alcanzar flujos arteriales altos ${ }^{1,5}$.

La HDF post-dilucional, debido al método de infusión tras el dializador, permite obtener aclaramientos de pequeños solutos y de sustancias de mayor peso molecular.
Este método puede provocar complicaciones técnicas, como la hemoconcentración o elevación de la presión transmembrana (flujos de ultrafiltración limitados). Para conseguir un volumen adecuado de recambio, el flujo de sangre debería ser superior a $350 \mathrm{ml} / \mathrm{min}$, lo que requiere de accesos vasculares que permitan altos flujos de sangre ${ }^{1,5}$. Desde que Ahrenholz y cols en 1997 determinaron que la HDF post-dilucional era más eficaz que la pre-dilucional, llevado a cabo con el mismo volumen de infusión, se considera a la HDF post-dilucional como la técnica más eficaz ${ }^{6}$. Sin embargo, un reciente estudio pone en cuestión esta convicción ${ }^{7}$.

El objetivo de este estudio fue comparar distintos parámetros dialíticos y hemodinámicos entre los modos de HDF pre y post-dilucional.

\section{Material y método}

Se realizó un estudio prospectivo transversal en una población en diálisis que ya se trataba con HDF en línea. Los pacientes fueron sometidos durante 4 semanas a cada alternativa de HDF: pre y post-dilucional ( 12 sesiones en cada modalidad).

Durante el estudio se registraron los valores de tensión arterial sistólica, diastólica y frecuencia cardiaca pre y post hemodiálisis, flujo de sangre, presión venosa, volumen de sangre dializado y volumen de sustitución. La dosis de diálisis se midió mediante dialisancia iónica (módulo OCM).

Se utilizó la misma membrana (Helixona $1,8 \mathrm{~m}^{2}$ ), misma dosis de heparina, mismo tiempo de diálisis en las dos técnicas a estudio.

El tratamiento se realizó con monitores $5008^{\circledR}$, cuya tasa de sustitución se seleccionó en modo autosustitución tanto para la pre como para la post-dilucional, y monitores $4008 \mathrm{~S}^{\circledR}$, cuya tasa de sustitución se seleccionó en un $25 \%$ del flujo de sangre durante el periodo post-dilucional y en un $33 \%$ del flujo de sangre durante el periodo pre-dilucional.

Los datos recogidos fueron analizados mediante el paquete estadístico SPSS versión 15.0 para Windows. Se realizó un análisis descriptivo de las variables recogidas, y para la inferencia estadística se utilizó el test de los 
rangos de Wilcoxon. Los resultados se consideraron significativos si el nivel crítico observado era inferior al $5 \%(p<0,05)$.

\section{Resultados}

Se incluyeron en la muestra del estudio 26 pacientes: $30 \%$ mujeres y $70 \%$ hombres, $61 \pm 13$ años. El tiempo medio en tratamiento renal sustitutivo fue de $117 \pm 124,45$ meses, tiempo medio en hemodiálisis fue de $50 \pm 54,38$ meses. La etiología de la insuficiencia renal más prevalente fue la vascular y la glomeru$\operatorname{lar}(26,9 \%)$, seguida de otras $(23,1 \%)$, la sistémica (19,2\%) y la intersticial (3,8\%).El 57,7\% tenía como acceso vascular una FAVI y el $42,3 \%$ un catéter.

Los resultados de las distintas determinaciones se representan en la tabla 1 :

Los parámetros de tensión sistólica, diastólica, frecuencia cardiaca, flujo de sangre, presión venosa y volumen de sangre dializado no presentan diferencias significativas entre las dos alternativas de HDF pre y post-dilucional. Se obtiene un mayor valor de KT estadísticamente significativo en la técnica de HDF post-dilucional precisando ésta de un volumen de sustitución de la mitad, en comparación con la HDF pre-dilucional.

\section{Discusión}

Los métodos actuales de la diálisis HDF en línea más utilizados son la HDF post y pre-dilución y la milddilucional, que combina ambas técnicas, presentando ambos tanto características positivas como negativas. Según varios estudios ${ }^{6,8-10}$, la HDF post-dilucional es el método de infusión más eficiente para obtener aclaramientos de pequeños solutos y sustancias de mayor peso molecular, con el inconveniente de la hemoconcentración, la HDF pre-dilucional permite mejor extracción de medias y altas moléculas y aclaramientos más bajos de solutos de pequeño peso molecular, reduciendo la hemoconcentración, y la mild-dilucional es la técnica que al combinar las anteriormente citadas elude las desventajas de ambas. Fernández López y cols evaluaron la eficacia dialítica entre HDF pre y post dilucional, en igualdad de flujo de sustitución, en 20 pacientes. Concluyeron que cuando comparaban HDF on-line post frente a pre-dilucional en igualdad de volúmenes, obtenían que la post-dilucional es la técnica que obtiene mejor eliminación de solutos urémicos. Sin embargo, cuando comparaban ambas técnicas en igualdad de presiones, la HDF pre-dilucional era la que obtenía mayor eficacia, como consecuencia de la desigualdad en los volúmenes de infusión. Cuando comparaban la eficacia de ambas técnicas a máxima eliminación de solutos, era la HDF pre-dilucional la que obtenía mayor eliminación de solutos ${ }^{7}$.

\begin{tabular}{|l|c|c|c|}
\hline & $\begin{array}{c}\text { HDF post } \\
\text { dilucional }\end{array}$ & $\begin{array}{c}\text { HDF pre } \\
\text { dilucional }\end{array}$ \\
\hline Tensión arterial sistólica pre HD (mmHg) & $122,05 \pm 36,03$ & $123 \pm 35,78$ \\
\hline Tensión arterial diastólica pre HD (mmHg) & $67,74 \pm 16,28$ & $67,99 \pm 15,05$ & 0,672 \\
\hline Tensión arterial sistólica post HD (mmHg) & $115,14 \pm 29,7$ & $115,65 \pm 32,96$ & 0,715 \\
\hline Tensión arterial diastólica post HD (mmHg) & $64,83 \pm 35,44$ & $63,6 \pm 14,91$ & 0,550 \\
\hline Frecuencia cardiaca pre HD (I.p.m.) & $73,83 \pm 11,55$ & $75,36 \pm 38,44$ & 0,534 \\
\hline Frecuencia cardiaca post HD (I.p.m.) & $75,41 \pm 12,33$ & $74,41 \pm 12,1$ & 0,588 \\
\hline Flujo de sangre (mI/min) & $322,57 \pm 29,93$ & $323,35 \pm 27,55$ & 0,972 \\
\hline Presión venosa (mmHg) & $166,07 \pm 32,1$ & $170 \pm 29,87$ & 0,084 \\
\hline Volumen de sangre (I) & $71,33 \pm 10,29$ & $71,07 \pm 7,25$ & 0,301 \\
\hline Volumen de sustitución (I) & $16,65 \pm 3,2$ & $30,25 \pm 11,36$ \\
\hline Kt (I) & $50,32 \pm 7,35$ & $49,02 \pm 22,34$ & $<0,001$ \\
\hline
\end{tabular}

Tabla 1. Valores medios \pm desviación típica de los parámetros hemodinámicos y dialíticos estudiados según modalidad de HDF, junto con la significación estadística 
El objeto de nuestro estudio se ha basado en el análisis de parámetros hemodinámicos y dialíticos, no siendo en este caso valorado el aclaramiento de moléculas, donde se encuentra mayor referencia bibliográfica.

En cuanto al trabajo de enfermería no se ha objetivado mayor número de incidencias entre una técnica u otra. Los parámetros hemodinámicos no mostraron diferencias que nos hagan decantarnos por una de las dos alternativas de tratamiento. Conseguimos similares flujos de sangre, presión venosa $y$ volumen de sangre dializado tanto en pre como en post-dilucional.

Se obtienen mejores resultados de KT y con menor variabilidad en la técnica de hemodiálisis post-dilucional, destacando como dato importante la menor necesidad de aporte de líquido de sustitución en esta alternativa, siendo la mitad que en pre-dilución. Dato más importante a reseñar en este estudio, dado que el gasto de agua ultrapura necesaria para lograr resultados incluso mejores de $\mathrm{KT}$, se reduce al $50 \%$ en la HDF post-dilucional.

Si bien nos encontramos con pacientes de mayor edad y con peores accesos vasculares que en la mayoría de las ocasiones no permiten alcanzar flujos elevados como requiere la técnica post-dilucional, es por ello que para estos casos se podría considerar como técnica de elección la pre-dilucional.

Creemos que la dosis de heparina en el modo predilucional debe ser menor, pero no ha sido objeto de este estudio y no se ha modificado la pauta de la misma en ninguna de las técnicas.

Destacar que los parámetros hemodinámicos no muestran diferencias significativas. La técnica de HDF es bien tolerada en las dos modalidades de infusión.

Ante un mismo flujo de sangre, presión venosa y volumen de sangre dializado se consigue una mejor dosis de diálisis en el modo post-dilucional.

La HDF post-dilucional parece ser una mejor alternativa respecto a la pre-dilucional en relación a los parámetros estudiados siempre que no se tenga en cuenta la problemática de los accesos vasculares.
Con dicha técnica logramos mejores resultados de KT precisando la mitad de volumen de sustitución, con el consiguiente ahorro de agua ultrapura.

La HDF pre-dilucional puede ser una alternativa para aquellos pacientes que no se consiga un flujo arterial alto.

Dejamos pendiente para futuros trabajos el estudio de otros parámetros que no se han incluido en el actual y que podrían aportarnos resultados relevantes.

Está por estudiar a más largo plazo las ventajas e inconvenientes de la HDF de alto flujo.

Recibido: 24 Noviembre 2011

Revisado: 30 Noviembre 2011

Modificado: 10 Enero 2012

Aceptado: 20 Enero 2012

\section{Bibliografía}

1. Sánchez $M$, Vallvé $M R$, López $M T$, Gispert $N$, Mayordomo A, Lage S, Vives A. Comparación de hemodiafiltración "mid-dilucional" respecto a hemodiafiltración pre y postdilucional. Rev Soc Esp Enferm Nefrol 2009; 12 (1): 6-10.

2. Maduell F. Eficacia depurativa de medianas y grandes moléculas en diferentes modalidades de hemodiálisis.Nefrología,2005; 25 (Supl.2);15-18.

3. Puccini S, Crespo K. Hemodifiltración en línea: comparación de la eficacia pre postdilución. Libro de comunicaciones presentadas al XXVII Congreso Nacional de la Sociedad Española de Enfermería Nefrológica,2002: pp 113-118. En: SEDEN. Comunicaciones presentadas a los Congresos de la Sociedad Española de Enfermería Nefrológica 1981-2010 [CD-ROM]. Madrid: SEDEN; 2010.

4. Castañeda C, Ciriza A y Diez R.Hemodiafiltración en línea en 52 pacientes: evolución, clínica y analítica. Rev Soc Esp Enferm Nefro.2005; 8(3). 
5. Fernández $M$, Teruel JL. Técnicas de hemodiálisis. En: Lorenzo V, Lopez-Gomez J.M, Martin de Francisco AL, Hernandez D. Nefrología al día. Madrid: Grupo Editorial Nefrología de la Sociedad Española de Nefrología; 2010. p.437-445.

6. Ahrenholz $P$, Winkler $E$, Ramlow $W$, Tiess $M$, MüIler W. Online haemodiafiltration with pre- and postdilution: a comparison of efficacy. Int $\mathrm{J}$ Artif Organs.1997; 20: 81-90.

7. Fernández López $P$, Peña Ortega $M$, Mañero Rodríguez C, Polo Moyano A, Palma Barrio R, Borrego García $E$ y cols. ¿Es la hemodiafiltración on-line posdilucional más eficaz que la predilucional?: estudio en isovolumetría frente a isobarometría. Nefrologia 2010; 30(Supl 1): 71.
8. Maduell F, García H, Hdez.-Jaras J, Calvo C, Navarro V. Comparación de la infusión predilucional versus postdilucional en HDF en línea. Nefrología 1998;18 (Supl. 3): 49.

9. Fernández AV, Soto S, Arenas M, Horrilo F, Sáez N, Pérez L. Comparación de infusión automatica respecto a manual en hemodiafiltración on-line post dilucional. Rev Soc Esp Enferm Nefrol 2010; 13 (1):17-22.

10. Rabindranath KS, Strippoli GF, Daly C, Roderick PJ, Wallace S, MacLeod AM. Haemodiafiltration, haemofiltration and haemodialysis for end-stage kidney disease. Cochrane Database Syst Rev 2006;18:CD006258. 